\title{
Froissart bound, diffraction scattering of hadrons, and scaling at asymptotic energies
}

\author{
Aruna Kumar Nayak $\odot^{*}$ and Jnanadeva Maharana $\odot^{\dagger}$ \\ Institute of Physics, Sachivalaya Marg, Bhubaneswar 751005, India
}

(Received 11 May 2020; accepted 28 July 2020; published 14 August 2020)

\begin{abstract}
The Froissart bound on the total cross section, $\sigma_{t}$, is subjected to test against very high energy data. We have found no clear evidence for its violation. The scaling property of the differential cross section in the diffraction region is investigated. It exhibits scaling in the ISR, SPS, Tevatron, and LHC energy domain which had hitherto remained unexplored. The slope of the diffraction peak is fitted and the data are tested against the rigorous bounds.
\end{abstract}

DOI: 10.1103/PhysRevD.102.034018

\section{INTRODUCTION}

The high energy scattering of hadrons is of great interest. The total cross sections $\sigma_{t}^{p p}$ and $\sigma_{t}^{p \bar{p}}$, from ISR to LHC energies, are rising and the cosmic ray data continues to follow the trend. The elastic cross sections, $\sigma_{e l}$, grow with energy as well. The high energy scattering data are endowed with several fascinating features [1]. The elastic differential cross sections exhibit forward peak whose width shrinks as energy grows. The slope of the diffraction peak increases with energy. There are phenomenological models to study high energy hadronic processes. There is another approach to study strong interactions without appealing to any specific model. It is the S-matrix formulation pioneered by Heisenberg [2]. This philosophy, over the years, has evolved into axiomatic field theory approach as known at present. The rigorous results derived in this framework are not based on any model. Indeed, the structure rests on certain fundamental axioms to be respected by relativistic quantum field theories (QFT). The analyticity properties and other attributes of scattering amplitudes are proved starting from the axioms. The hallmark of the axiomatic formulation is that the principal results are presented as upper and lower bounds on experimentally measured quantities. So far there are no experimental evidences for the violation of any of these bounds. One of the most celebrated results is the FroissartMartin bound on the total hadronic cross sections, $\sigma_{t}$. Throughout this paper the high energy data means the data

\footnotetext{
nayak@iopb.res.in

†maharana@iopb.res.in
}

Published by the American Physical Society under the terms of the Creative Commons Attribution 4.0 International license. Further distribution of this work must maintain attribution to the author(s) and the published article's title, journal citation, and DOI. Funded by SCOAP. accumulated in the energy range covered by accelerators such as ISR, SPS, Tevatron, and LHC. Whenever the data of cosmic rays are incorporated they will be referred to explicitly. The high energy accelerators have been accumulating data over the years. It is worthwhile to focus attention on such data and study afresh how they put the bounds to tests.

The purpose of this article is to investigate certain areas of strong interaction processes which have remained unexplored in the high energy regime so far. We shall consider three topics as mentioned below. (1) Our intent is to test the validity of the Froissart-Martin bound $[3,4]$

$$
\sigma_{t}(s) \leq \frac{4 \pi}{t_{0}} \log ^{2}\left(\frac{s}{s_{0}}\right)
$$

in the asymptotic energy regime. Here $s$ is the c.m. energy squared, $t_{0}$ is a constant less than or equal to the $t$-channel threshold. The prefactor in (1) was fixed by Martin [4] from the first principles. $s_{0}$ is introduced to make argument of the $\log ^{2}$ dimensionless and it cannot be determined $a b$ initio. However, recently, there has been attempts to determine $s_{0}$ with additional inputs [5]. The first important point to note is that the total cross section cannot have arbitrary energy dependence. Moreover, this bound is not obtained from any model in a perturbative framework, rather, it is a consequence of general axioms. Therefore, any experimental evidence for violation of the bound (1) would be a matter of concern. We have adopted a strategy to test the FroissartMartin bound in the very high energy regimes alluded to earlier. We shall outline our prescription in the next section. (2) The scaling of the differential cross section, $\frac{d \sigma}{d t}(s, t)$, in the diffraction region, i.e., in the near forward direction, is an interesting and intriguing feature in high energy reactions. This attribute has been observed in the pre-ISR energy domain in the past. The scaling, in the present 
context, is interpreted as follows. The scattering amplitude, $F(s, t)$, is a function of two Lorentz invariant variables $s$ and the momentum transferred squared, $t$. In the limit, $s \rightarrow \infty$, for fixed physical $t, F(s, t) \rightarrow f(\tau) ; f(\tau)$ being a function of a single variable. The mystery about such a scaling lies in the fact that perturbative QCD techniques cannot be applied to explain such an observed scaling. The problem is that in the kinematical region in question, i.e., large $s$ and small $|t|$, the reaction is soft; therefore, the power of the asymptotic freedom is inapplicable. We shall elaborate more on this type of scaling in sequel. In the past, several models [6-9] were proposed which described high energy scattering and investigated scaling. A good review of scaling phenomena with extensive references is presented in [9]. We examine scaling in the aforementioned energy domain. (3) The slope of the diffraction peak, $b(s)$, is a very important parameter in high energy hadronic collisions. It is not only useful to describe diffraction scattering but also it is utilized by the experimentalists to extract $\sigma_{t}$. The interests in energy dependence of $b(s)$ is to test the shrinking of the diffraction peak with energy; furthermore, axiomatic bounds would also be tested. Moreover, in the past, the measured diffraction peak data almost saturated the lower bound for the absorptive part of the differential cross section; leading to the conjecture that the amplitude is dominated by the imaginary part.

Remarks: (i) The analyticity of the 4-point scattering amplitudes is the cornerstone of the bound (1). The rigorous results such as analyticity and crossing symmetry are proved from axiomatic field theories [10-13]; either in the framework of Lehmann-Symanzyk-Zimmermann (LSZ) formulation [14] or from Wightman's axioms [15]. We recall the axioms of LSZ for sake of completeness. (a1) The states of the system are represented in a Hilbert space, $\mathcal{H}$. All the physical observables are self-adjoint operators in the Hilbert space, $\mathcal{H}$. (a2) The theory is invariant under inhomogeneous Lorentz transformations. (a3) The energy-momentum of the states are defined. It follows from the requirements of Lorentz and translation invariance that we can construct a representation of the orthochronous Lorentz group. The representation corresponds to unitary operators, $U(a, \Lambda)$, and the theory is invariant under these transformations. Thus there are Hermitian operators corresponding to spacetime translations, denoted as $P_{\mu}$, with $\mu=0,1,2,3$ which have following properties:

$$
\left[P_{\mu}, P_{\nu}\right]=0 .
$$

If $\mathcal{F}(x)$ is any Heisenberg operator then its commutator with $P_{\mu}$ is

$$
\left[P_{\mu}, \mathcal{F}(x)\right]=i \partial_{\mu} \mathcal{F}(x) .
$$

It is assumed that the operator does not explicitly depend on spacetime coordinates. If we choose a representation where the translation operators, $P_{\mu}$, are diagonal and the basis vectors $\mid p, \alpha>$ span the Hilbert space, $\mathcal{H}$,

$$
P_{\mu}|p, \alpha\rangle=p_{\mu}|p, \alpha\rangle
$$

then we are in a position to make more precise statements:

(a) Existence of the vacuum: there is a unique invariant vacuum state $|0\rangle$ which has the property

$$
U(a, \Lambda)|0\rangle=|0\rangle
$$

The vacuum is unique and is Poincare invariant.

(b) The eigenvalue of $P_{\mu}, p_{\mu}$, is lightlike, with $p_{0}>0$. We are discussing only the case of massive states. If we implement infinitesimal Poincaré transformation on the vacuum state then

$$
P_{\mu}|0\rangle=0, \quad \text { and } \quad M_{\mu \nu}|0\rangle=0
$$

from above postulates; note that $M_{\mu \nu}$ are the generators of Lorentz transformations. (a4) The locality of theory implies that a (bosonic) local operator at spacetime point $x^{\mu}$ commutes with another (bosonic) local operator at $x^{\prime \mu}$ when their separation is spacelike i.e., if $\left(x-x^{\prime}\right)^{2}<0$. Our Minkowski metric convention is $(1,-1,-1,-1)$. The fixed- $t$ dispersion relations for, $F(s, t)$, is proved from the above axiomatic theories and $t$ must lie within the LehmannMartin ellipse [16,17]. Moreover, there are several theorems, presented as upper and lower bounds which have stood the experimental tests so far [18-20]. (ii) The aforementioned scaling lies in a kinematical domain (large $s$ and small $|t|$ ) such that the perturbative QCD techniques cannot be applied to explain it. In the context of this scaling, the axiomatic field theory provides a precise and rigorous definition and proves the raison d'etre of the scaling [21]. We have chosen two different types of scaling variables to investigate how the data respond to our propositions. The choice is motivated under certain mathematical grounds and it is not of purely phenomenological as we shall explain later. (iii) The combined data of $\sigma_{t}^{p p}$ and $\sigma_{t}^{p \bar{p}}$ are used to test the validity of Froissart bound. The point to note is that the rising of $\sigma_{t}^{p p}$ and $\sigma_{t}^{p \bar{p}}$ was first observed at ISR. It was noted that $\Delta \sigma=\sigma_{t}^{p p}-\sigma_{t}^{p \bar{p}}$ is small and it decreases with growing ISR energy. The Pomeranchuk's theorem [22-24] provides a clue for us from the above observed feature. The original theorem stated that the particle-particle and the particle-antiparticle total cross sections would tend to equal values asymptotically; however, an important assumption was that [22] total cross sections attain constant values at asymptotic energies. We recall that $\sigma_{t}^{p p}$ and $\sigma_{t}^{p \bar{p}}$ continue to rise at ISR, SPS, Tevatron and LHC energies; cosmic ray confirms the same trend. Note that the Pomeranchuk theorem is not a consequence of axioms of the QFT. The generalized theorem for the case of rising cross sections has been proved [25]. We recall that only ISR has measured the two cross sections, $\sigma_{t}^{p p}$ 
and $\sigma_{t}^{p \bar{p}}$, at the same energy and therefore, the theorem can be tested there. When fits to $p p$ and $p \bar{p}$ cross section data are extrapolated, the two curves show a tendency to converge asymptotically. We justify, therefore, to combine the two sets of data and fit the cross sections.

The article is organized as follows. The next section, Sec. II, is devoted to study of phenomena mentioned earlier. In Sec. II A, we propose a formula to fit the combined total cross section data, $\sigma^{p p}$ and $\sigma_{t}^{p \bar{p}}$, from ISR energies all the way up to the cosmic rays. We present a justification for the choice of the fitting formula. The study of the scaling of the elastic differential cross section is carried out in Sec. II B. We briefly recall the essential results on this type of scaling envisaged from rigorous mathematical structure. The results from the axiomatic approach are utilized for our purpose. We examine the scaling of diffractive elastic scattering. We choose two different scaling variables to investigate this phenomenon. Section II C is devoted to the study of the slope of the diffraction peak, $b(s)$. As we have noted earlier, it is an important experimentally measured parameter. We mention in passing that, to our surprise, there has not been much activity to explore the energy dependence of $b(s)$. We fit $b(s)$ from ISR energies to the LHC energy. Moreover, there are rigorous bounds on the slope of the absorptive diffraction differential cross sections, $b^{A}(s)$. There is no strong reason that why the data should respect these bounds. The motivation lies in the fact that, in the past, the data seemed to saturate the lower bound which led to an interesting conjecture. We test the bounds against the data. The third section contains the summary of our results and conclusions.

\section{ANALYSIS OF EXPERIMENTAL DATA}

We proceed to fit $\sigma_{t}$, investigate the scaling of differential cross sections, and the energy dependence of the slope parameter.

\section{A. The fit to total cross sections}

The purpose is to fit the total cross section data in order to test the Froissart-Martin bound. We would like to draw attention to the following facts. The PDG [1] has presented a very good fit to total cross sections of a large number of hadronic reactions over a wide range of energies. Let us discuss their fit to $\sigma_{t}^{p p}$. They considered data from $5 \mathrm{GeV}$ all the way up to LHC energies; moreover, the cosmic ray data are also incorporated in the fit. We recall that the Froissartbound saturating energy dependence is incorporated in their fit. In other words, the term is $\log ^{2} \frac{s}{s_{0}}$. The coefficient of this term, the Heisenberg constant, $H$, and $s_{0}$ are treated as floating parameters. In addition there is a constant term, $\mathrm{P}$, associated with Pomeranchuk trajectory and several other terms corresponding to the contributions of Regge trajectories were added. It is important to note that the total cross sections are flat from $5 \mathrm{GeV}$ till the pre-ISR energy domain and there are vast data points measured with very good precision. Moreover, in the energy interval noted above, the Regge trajectory, such as $\rho, A_{2}$.. contributions are important although they fall off as power of $s$ compared to the Pomeranchuk term and the Heisenberg term. In the fit to $\sigma_{t}$, the vast set of data points where $\sigma_{t}$ is constant, terms subdominant compared to $\log ^{2} s$ term, play a crucial role. Moreover, the fit does not set out to test the FroissartMartin bound since its exponent is fixed as 2. Our objective is to test the bound; as a consequence, we take the power of the exponent to be a floating parameter. A point is in order. It was claimed, in the past, in a fit to $\sigma_{t}^{p p}$, that there is evidence for violation of the Froissart bound [26]. Subsequently this claim was refuted by Block and Halzen [27]. Note that those two papers covered the same energy range as was covered by the PDG [1], i.e., from $5 \mathrm{GeV}$ to cosmic ray energies. There is a recent comprehensive review [28] on high energy collisions which covers diverse aspects of hadronic processes and we refer it to the interested reader.

Let us discuss our proposal to fit the total cross section data. We choose the following parametrization to fit the combined data.

$$
\sigma_{t}=H \log ^{\alpha}\left(\frac{s}{s_{o}}\right)+P .
$$

$H$ and $P$ are the Heisenberg and Pomeranchuk constants, respectively. $P$ is the contribution of the Pomeranchuk trajectory in the Regge pole parlance. The constants $H, P$ and $\alpha$, are free parameters and are determined from the fits. We fix $s_{0}=16.00 \mathrm{GeV}^{2}$, taking a hint from the PDG fit. PDG adopted the following strategy to fit $\sigma_{t}$ data. For the fit to $\sigma_{t}^{p p}$ the chosen energy range was from $5 \mathrm{GeV}$ to cosmic ray regime. The Froissart-bound-saturating energy dependence is incorporated in their fitting procedure. Note that in the pre-ISR energy regime the measured cross sections are flat and measured with very good precision. Moreover, Regge pole contributions, with subleading power behaviors, should be included in the pre-ISR energy domain. However, in the energy range starting from ISR, the Regge contributions are negligible. It is worth while to discuss and justify the reasons for not including the contributions of subleading Regge poles to $\sigma_{t}$ in the energy range starting from ISR point and beyond (where our interests lie). Moreover, the subleading Regge poles have an important roles to play in fitting $\sigma_{t}$ where the data are collected from the accelerators of those period. We refer to [18] and to the review article of Leader [29] for detailed discussions. We recall that $\sigma_{t}$ is almost constant in the "low energy" (preISR) region, however, contribution of the Regge tail is also necessary. Let us consider the case of $p p$ scattering to get a concrete idea. The Pomeranchuk trajectory contributes a constant term to $\sigma_{t}$ and its intercept is $\alpha_{P}(0)=1$. Then there are subleading trajectories corresponding to $\omega, \rho, A_{2}$, 
$\phi$, etc. When a fit to $\sigma_{t}^{p p}$ was considered by Rarita et al. [30], they concluded, from numerical fits, that the $\omega$ trajectory dominates $[29,30]$ and the contributions of other Regge trajectories is quite small [31]. They found that the Regge residue (interpreted as the Regge trajectory coupling) is $R_{p p \omega} \approx 15.5 \mathrm{mb}$ and $\alpha_{\omega}(0) \approx 0.45$ and the Regge scale, to define a dimensionless ratio (say $\frac{s}{s_{*}}$ ) is $s_{*}=1 \mathrm{GeV}^{2}$. Let us estimate what is the contribution of the $\omega$-trajectory to $\sigma_{t}^{p p}$ at the ISR energy. The contribution of the $\omega$-trajectory to $\sigma_{t}$ is quite small in the energy range from ISR to LHC. For example, at ISR energy of $\sqrt{s}=23.5 \mathrm{GeV}$, the $\omega$-Regge pole contribution to $\sigma_{t}$ is approximately $0.5 \mathrm{mb}$ whereas at LHC, for $\sqrt{s}=8 \mathrm{TeV}$, it is $\approx 0.001 \mathrm{mb}$; the corresponding $\sigma_{t}$ are $\approx 39 \mathrm{mb}$ and $\approx 103 \mathrm{mb}$ at $23.5 \mathrm{GeV}$ and $8 \mathrm{TeV}$ respectively. The parametrizations of [30] is used for the above estimates. Consequently, for our purpose, the parametrization (7) is well justified.

We considered the combined data of $\sigma_{t}^{p p}$ and $\sigma_{t}^{p \bar{p}}$ for the energy range as mentioned earlier. ISR data are from $\sqrt{s}=$ 23.5 to $63 \mathrm{GeV}$ [32-41]. SPS data are at 540, 541, 546, and $900 \mathrm{GeV}$ [42-48], whereas Tevatron data points are at $546 \mathrm{GeV}, 1.02 \mathrm{TeV}$, and $1.8 \mathrm{TeV}$ [49-56]. The LHC data points are at 7, 8 and $13 \mathrm{TeV}$ [57-64]. Cosmic ray points are at $8,14,24,30,57$, and $95 \mathrm{TeV}$ [65-68]. The measured values of cross sections against $\sqrt{s}$, along with the fitted curve, are shown in Fig. 1. The fitted values for the parameters are $P=36.4 \pm 0.3 \mathrm{mb}, H=0.22 \pm 0.02 \mathrm{mb}$, and $\alpha=2.07 \pm 0.04$. The quality of the fit, as reflected by the $\chi^{2} /$ n.d.f. is found to be moderate due to inclusion of both $\sigma_{t}^{p p}$ and $\sigma_{t}^{p \bar{p}}$ measurements from ISR. A fit excluding $\sigma_{t}^{p \bar{p}}$ from ISR, as shown in Fig. 1 (lower), improves the fit quality without significantly changing the value of the fit parameters. We find no conclusive evidence for the violation of the Froissart bound.

\section{B. Scaling of the diffraction cross section}

The scaling observed in the diffraction region is an unexpected feature. This scaling phenomena is mysterious in the sense that we are unable to explain it from perturbative QCD perspectives. The kinematical region of interests (i.e., large $s$ and small $|t|$ ) does not fall into the purview of the perturbative QCD terrain. Moreover, we draw attention to the fact that so far there is no careful analysis of the scaling in the diffraction region of very high energy regime which we intend to explore. Therefore, it deserves a thorough investigation. A precise definition of scaling and its derivation was provided from axiomatic field theory approach. The necessary ingredients stated and proved from the axiomatic field theory are [21]: (i) The scattering amplitude, $F(s, t)$, is holomorphic in the disk $|t|<t_{0}$ for any $s$ in cut s plane, where $t_{0}$ is a constant less than or equal to the $t$-channel threshold. (ii) The polynomial boundedness: $|F(s, t)|$ is bounded by $s^{N}, N \in Z$ and $\mathrm{N}$ is

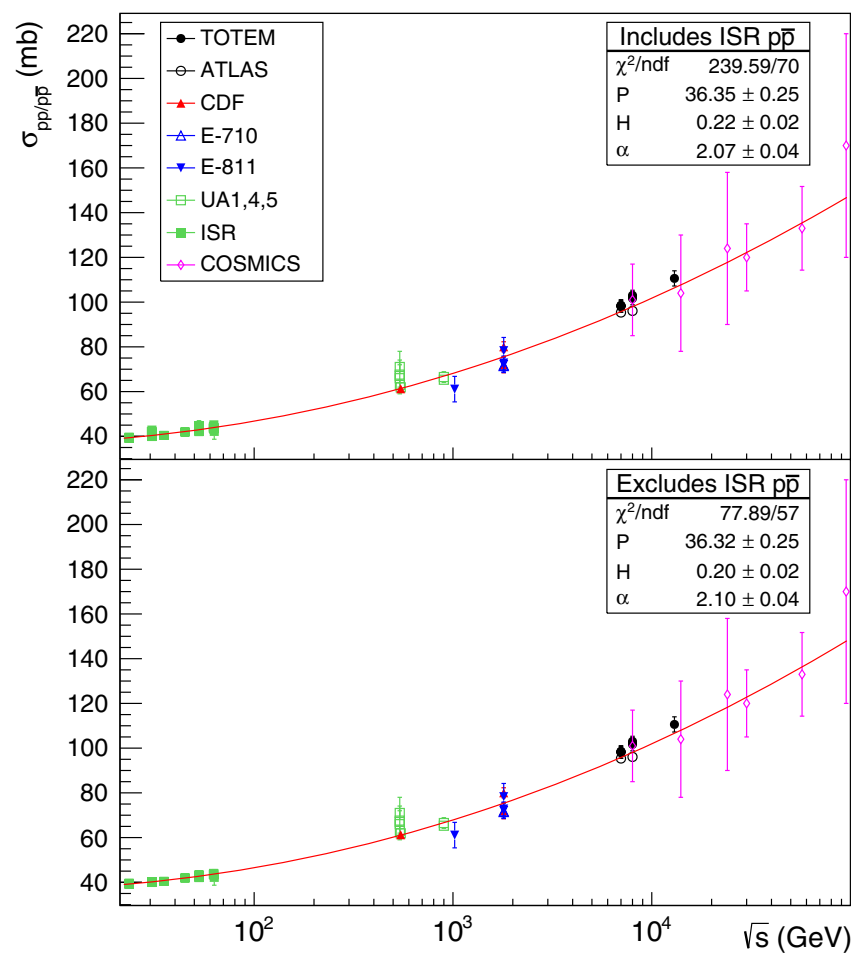

FIG. 1. $\sigma_{t}^{p p}$ and $\sigma_{t}^{p \bar{p}}$ against $\sqrt{s}$, measured by various experiments. The data points are fitted to a function defined in (7). The upper plot includes both $p p$ and $p \bar{p}$ data points, while the lower one excludes $p \bar{p}$ data points from ISR experiments.

finite, for $|t|<t_{0}$ and $s \rightarrow+\infty$. (iii) $F(s, t)$ satisfies unitarity in the s-channel. It follows from the results (i)-(iii) that $F(s, t)$ satisfies the bound

$$
|F(s, t)| \leq\left[\frac{4 \pi \sigma_{e l}(s)}{t_{0}}\right]^{1 / 2} s \log (s) \exp \left[\left(\frac{|t|}{t_{0}}\right)^{1 / 2} \log (s)\right] .
$$

The Pomeranchuk-theorem-violating amplitude (see [21]) asymptotically behaves as

$F(s, 0) \sim i \sigma s+C s \log (s), \quad 0<|C|<\left(4 \pi \sigma / t_{0}\right)^{1 / 2}$

where $\sigma$ is the total cross section. There is a more refined mathematical statement on the behavior of $F(s, 0)$ in [21]. A general class of amplitudes of the form

$$
F(s, 0) \sim(i \alpha+\beta) s(\log (s))^{-2}
$$

was considered by [21]; where $0<\alpha \leq\left(\frac{4 \pi}{t_{0}}\right),|\beta| \leq\left(\frac{4 \pi \alpha}{t_{0}}\right)^{1 / 2}$ and $F(s, 0)$ respects the Froissart bound. Our sole interest is to recapitulate the scaling property succinctly derived and proved in [21]. A function

$$
f(s, \tau)=\frac{F\left(s,-t_{0} \tau(\log (s))^{-2}\right)}{F(s, 0)}
$$


was introduced in [21]. The scaling is interpreted in the following sense: define $f(\tau)=\lim _{s \rightarrow \infty} f(s, \tau)$. It was proved that $f(\tau)$ is an entire function with following properties

$$
f(0)=1, \quad|f(\tau)|<\left(\frac{4 \pi C_{0}}{t_{0}}\right)^{(1 / 2)} e^{\sqrt{\tau}}, \text { for all } \tau
$$

where $C_{0}=\frac{\alpha}{\alpha^{2}+\beta^{2}} ; \alpha$ and $\beta$ are as in (10). In a nutshell, it summarizes the scaling phenomena as derived from the axiomatic perspectives.

We have chosen two different types of scaling variables based on certain theoretical consideration. (a) The first choice is $\tau_{1}=\frac{1}{16 \pi} \frac{\sigma_{t}^{2}}{\sigma_{e l}} t$. The technique of group contraction was adopted to describe high energy diffraction scattering [69]. It was demonstrated that the scaling occurs in the small $|t|$ domain at high energies. The high energy collision of spinless particles, in small angles, was envisaged. Let us consider a sphere of radius $\gamma$ with polar angles $\theta$ and $\phi$. The amplitude, for scattering of scalars, depends only on $\theta$. Let us focus on scattering in the forward regions such that $\gamma \theta \rightarrow$ finite as $\theta \rightarrow 0$ and $\gamma \rightarrow \infty$. The group contraction technique [70] was invoked as follows. $O(3)$ is the little group of the Lorentz group $O(3,1)$. In the limit, $\gamma \rightarrow \infty$, $O(3)$ contracts to $E_{2}$, the group of translations. Let $L_{i}, i=1,2,3$ be generators of $O(3)$ satisfying $\left[L_{i}, L_{j}\right]=i \epsilon_{i j k} L_{k}$. Define $L_{2}=\gamma Q_{2}$ and $L_{1}=-\gamma Q_{1}$ with $L_{3}$ unaltered. The angular momentum algebra assumes the form $\left[L_{3}, Q_{2}\right]=i Q_{1},\left[L_{3}, Q_{1}\right]=-i Q_{2}$ and $\left[Q_{1}, Q_{2}\right]=0$ in the limit $\gamma \rightarrow \infty$. Notice that $Q_{1}, Q_{2}$ are translation operators of $E_{2} . L_{3}$ generates rotation around the 3rd axis. If $q_{a}$ and $q_{b}$ are the momenta defining a representation of $E_{2}$, then the eigenvalue of Casimir of $O(3), l(l+1) \rightarrow$ $(l+1 / 2)^{2}$ in the limit $\gamma \rightarrow \infty$. The partial wave amplitude, $f_{l}(s) \rightarrow f(q, s)$ where $q=\sqrt{\left(q_{1}^{2}+q_{2}^{2}\right)}$. The expression for the scattering amplitude [69] is derived from the following ingredients: (i) Partial wave unitarity. (ii) Optical theorem to relate the absorptive forward scattering amplitude to $\sigma_{t}$. And (iii) utilize the standard relation $\sigma_{e l}=\frac{4 \pi}{p^{2}} \sum_{0}^{\infty}(2 l+1)\left|f_{l}(s)\right|^{2}, \quad p$ being the c.m. momentum. Next step is to replace $f_{l}(s)$ by $f(q, s)$ and the sum over $l$ goes over to an integral in $q$. Moreover, $f(q, s)$ is assumed to have a Gaussian distribution in $q$. Finally, the normalized differential cross section, after a few algebraic steps, was derived to be

$$
\frac{d \sigma}{d t}=\left.\frac{d \sigma}{d t}\right|_{t=0} e^{\frac{\sigma_{t}^{2}}{16 \pi \sigma_{e l}}}
$$

The identification of $\tau_{1}=|t| \frac{\sigma_{t}^{2}}{16 \pi \sigma_{e l}}$ as a scaling variable was noted in [69]. Indeed the diffraction scattering data, available at that juncture, for $\pi^{ \pm} p, k^{ \pm} p, p p$ and $p \bar{p}$ over a wide energy range exhibited the scaling behavior. The objective is to examine whether the present data exhibits the scaling. (b) The other choice is $\tau_{2}=|t| b(s)$, where $b(s)$ is the experimentally measured slope parameter. Cornille and Martin [71] have demonstrated rigorously the existence of this scaling variable. The data prior to SPS, Tevatron, LHC era exhibited the scaling [71]. Subsequent works [72-75] investigated and tested the scaling with the vast data of that era. Notice that the data in the forward direction is conveniently parametrized as

$$
\frac{d \sigma}{d t}(s, t)=\left.\frac{d \sigma}{d t}\right|_{t=0} e^{B(s, t)}
$$

where $B(s, t)$ is usually expanded in a power series in $t$, around $t=0$, as $B(s, t)=b(s) t+c(s) t^{2}+\ldots$; where $b(s)$ is the slope of the diffraction peak, $c(s)$ is the curvature and so on. Thus the $b(s) t$ term dominates in the small $t$, near forward region, while fitting $\frac{d \sigma}{d t}$. We restrict the values of $|t|$ to the range that is used in the corresponding measurements to extract the values of $b(s)$ assuming a purely exponential dependence of $\frac{d \sigma}{d t}$ on $t$. If we include larger values of $|t|$ then the data favor the retention of the higher curvature terms in the expansion of $B(s, t)$ in (14).

We have displayed scaling in the diffraction region for the choice $\tau_{1}=\frac{|t| \sigma_{t}^{2}}{16 \pi \sigma_{e l}}$ and $\tau_{2}=|t| b(s)$, in Fig. 2. We use $|t|$ in our definition of scaling variables for convenience $(t$ is always negative in the physical region for s-channel reactions). We have not included a set of ISR data points in a very small $|t|$ region; this point is discussed in detail in the context of extraction of $b(s)$ in sequel. If those points are included in the scaling plot they deviate from the scaling displayed by rest of the data. It is important to note that the rest of the data exhibits scaling so nicely from ISR to LHC energy range. Now let us compare and contrast the scaling phenomena with the two scaling variables. For the purpose of examining the scaling for the measured values of $d \sigma / d t$ as function of $|t|$ data are taken from $[42,57,59,76-83]$. We analyze the scaling property with $\tau_{1}=\frac{1}{16 \pi} \frac{\sigma_{t}^{2}}{\sigma_{e l}}|t|$ as the scaling variable and the covered range of $\tau_{1}$ is: $0.02 \leq \tau_{1} \leq 3.6$. The scaling is very good for the case of $\tau_{1}$ for small values of $|t|$ as depicted in Fig. 2(a). Note that the scaling is excellent for the $\tau_{2}$ variable in the small $|t|$ region and $0.05 \leq \tau_{2} \leq 9.0$, which is displayed in Fig. 2(b). There is a study of scaling in hadronic scattering [84]; however, their approach is different and their main focus is not in the kinematical domain of our interests.

\section{The slope of the diffraction peak}

The slope of the diffraction peak is defined to be

$$
b(s)=\left.\frac{d}{d t} \log \left(\frac{d \sigma}{d t}\right)\right|_{t=0}
$$



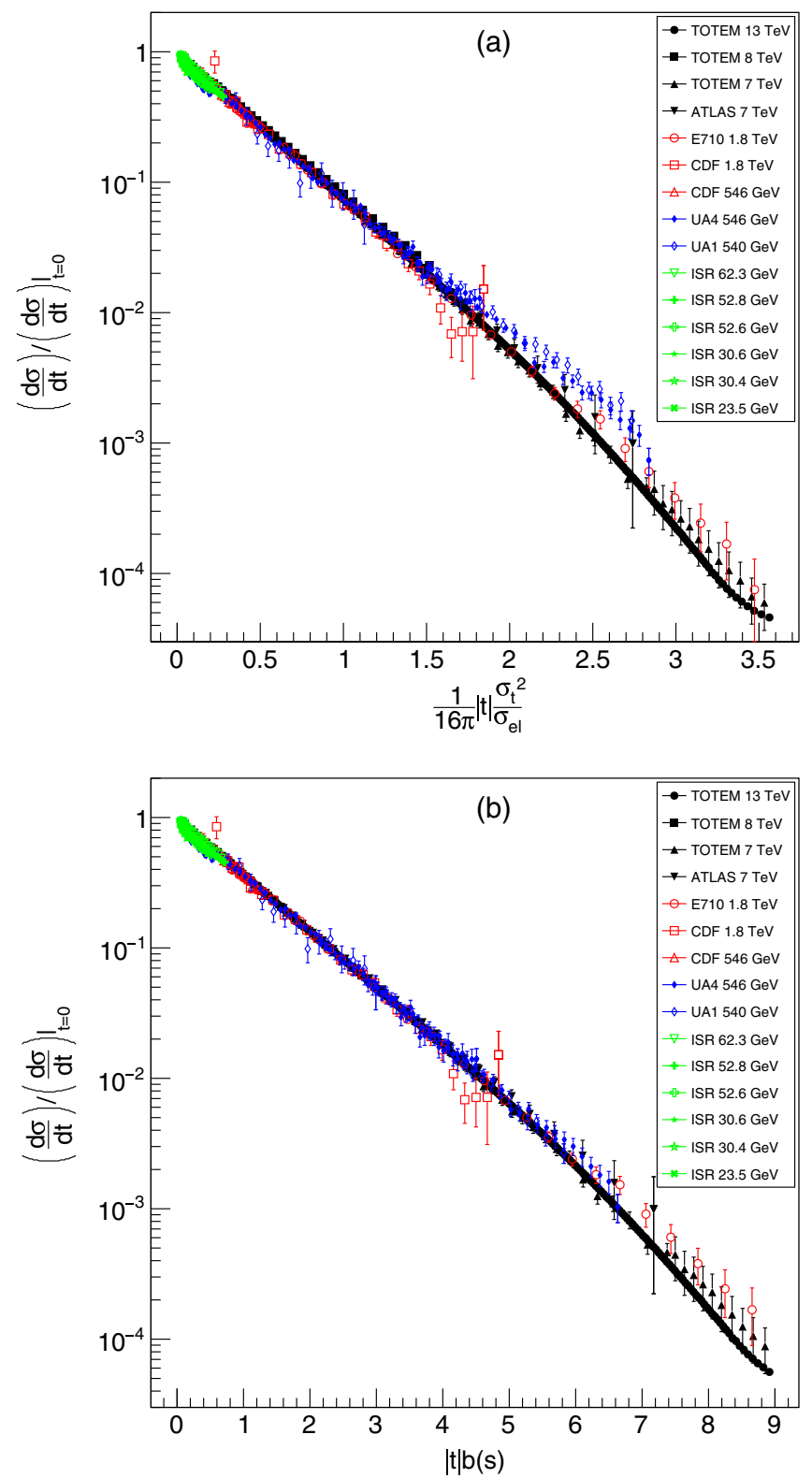

FIG. 2. Distributions of the ratio $\frac{d \sigma}{d t}(s, t) /\left.\frac{d \sigma}{d t}\right|_{t=0}$ as function of (a) $\frac{1}{16 \pi}|t| \frac{\sigma_{t}^{2}}{\sigma_{e l}}$ and (b) $|t| b(s)$, measured by various experiments for $p p$ and $p \bar{p}$ collisions.

and it is experimentally extracted from diffraction scattering data. Another object of interests is the slope of absorptive differential cross section

$$
b^{A}(s)=\left.\frac{d}{d t} \log \left(\frac{d \sigma^{A}}{d t}\right)\right|_{t=0} .
$$

There are rigorous lower and upper bounds on $b^{A}(s)$.

$$
\frac{2}{9}\left[\frac{\sigma_{t}^{2}}{4 \pi \sigma_{e l}}-\frac{1}{k^{2}}\right] \leq b^{A}(s) \leq \frac{1}{2\left(t_{0}-\epsilon\right)}\left[\log \left(\frac{s}{\sigma_{t}}\right)\right]^{2} .
$$

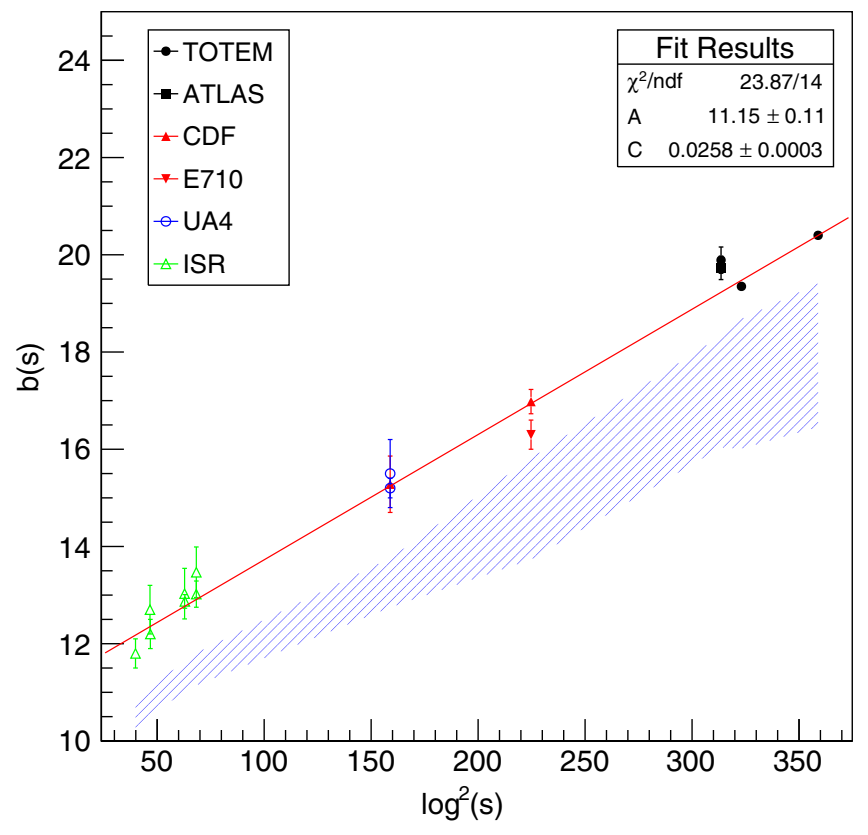

FIG. 3. Plot of measured value of $b(s)$ as function of $\log ^{2}(s)$ as obtained from various experiments. The error band is the lower limit as defined in (17).

The lower bound is due to Martin and MacDowell [85] whereas the upper bound was proved by Singh [86]. We have fitted the slope with the parameterization $b(s)=$ $A+C \log ^{2}\left(\frac{s}{s^{\prime}}\right)$ and chose $s^{\prime}=1 \mathrm{GeV}^{2}$ so that the argument of the $\log$ is dimensionless. The fit is depicted in Fig. 3. Note the following: the measurement of $\frac{d \sigma}{d t}(s, t)$ exhibits a sharp rise in the range $0.0004 \leq|t| \leq 0.004$. In this $|t|$ interval, $\frac{d \sigma}{d t}$ cannot be fitted by parametrizing it with only a $b(s)$. The experiment [76] fits the data by adding the proton form factor term to account for the Coulomb effect. Block and collaborators [87] consider an eikonal model to study pp scattering. They argue that in order to explain behavior of $\frac{d \sigma}{d t}$ in the said $|t|$ region, the effects of higher curvature terms be considered. We leave aside this set of data points of the particular ISR experiment to fit $b(s)$ taking into account the values of $b(s)$ as presented in various experiments. These experiments have extracted $b(s)$ from the fits to $\frac{d \sigma}{d t}$ in near forward direction. Let us examine how the measured $b(s)$ stands up against the bounds (17). We remind that the bounds (17) are proved for $b^{A}(s)$. Therefore, the data need not respect them. When the lower bound [85] was proved and was tested against experiments, the data nearly saturated the bound. Consequently, it was conjectured that the amplitude is dominantly imaginary [85]. In order to compare (experimentally) measured $b(s)$ against MacDowell-Martin bound we used measured values of $\sigma_{t}$ and $\sigma_{e l}$. Thus the bound depicted in Fig. 3 is a band due to the uncertainties of $\sigma_{t}$ and $\sigma_{e l}$ in measurements. The data satisfies the Martin-MacDowell bound, however. We remark that the upper bound (17) lies way above the 
data due to the presence of the factor $2 t_{0}, t_{0}=4 m_{\pi}^{2}$, in the denominator. The measured values of $b(s)$ are taken from $[42,57,59,76,79-83]$, which were used for the fit.

\section{SUMMARY AND CONCLUSIONS}

One of our objectives was to test the Froissart bound against experiments. We selected the set of data points where the total cross sections start rising. Since $\Delta \sigma$ is quite small at ISR energy and keeps diminishing with growing $s$ we justified the inclusion of both $p p$ and $p \bar{p}$ data in the fitting of $\sigma_{t}$. We set out to verify scaling of the diffraction data at asymptotic energies with small $|t|$. We note that this attribute of elastic scattering at asymptotic energy has hitherto escaped attentions and scrutiny. It is quite satisfying that the data exhibit very good scaling for both the choices: $\tau_{1}$ and $\tau_{2}$. The slope $b(s)$ is a very important parameter in the study of hadronic collisions and is used to extract $\sigma_{t}$ since the point $t=0$ is not accessed in experiments. We have presented a fit to $b(s)$ data. The axiomatic bounds of $b^{A}(s)$ was compared with the data, although the data need not necessarily respect them; however, they are consistent with the bounds.

There is an interesting paper on the ultra high energy neutrino-nucleon deep inelastic scattering [88]. In this work the authors study the behavior of the structure function. They show that the $\nu N$ scattering cross section exhibits $(\log (s))^{2}$ behavior.

The following remarks are in order. It is an important issue to ponder over the violation of the Froissart-Martin bound. So far the experimental data are consistent with the bound. If, in future, the experiments unambiguously confirm violation of the bound then it will be a matter of concern. One possible explanation would be to question the axioms of local field theories. However, these field theories do not lead only to the bound on total cross sections. There are other important inferences drawn from the axiomatic field theory. For example, the $C P T$ theorem [15] is another important outcome which has been put to tests. Therefore, it will not be so easy to abandon any of the axioms for local field theories. On the other hand, if we accept the axioms of local field theory, then new physics paradigms might have to be introduced. Thus observation of the violation of the bound on $\sigma_{t}$ would lead to very important consequences. In conclusion, we have examined several interesting and important aspects of very high energy scatterings and we have utilized the data to explore some of the phenomena which had not been explored in the recent years. We tested the validity of the sacred Froissart bound and it still stands.
[1] C. Patrignani et al. (Particle Data Group), Chin. Phys. C 40, 100001 (2016).

[2] W. Heisenberg, Z. Phys. 133, 65 (1952).

[3] M. Froissart, Phys. Rev. 123, 1053 (1961).

[4] A. Martin, Phys. Rev. 129, 1432 (1963).

[5] A. Martin and S. M. Roy, Phys. Rev. D 89, 045015 (2014).

[6] J. Dias de Deus, Nucl. Phys. B59, 231 (1973).

[7] A. J. Buras and J. Dias de Deus, Nucl. Phys. B71, 481 (1974).

[8] V. Barger, J. Luthe, and R. J. N. Phillips, Nucl. Phys. B88, 237 (1975).

[9] P. Valin, Phys. Rep. 203, 233 (1991).

[10] S. S. Schweber, Introduction to Relativistic Quantum Field Theory (Harper and Row, New York, 1961).

[11] R. Jost, General Theory of Quantized Fields (American Mathematical Society, Providence, 1965).

[12] R. F. Streater and A. S. Wightman, CPT Spin Statistics and All That (W. A. Benjamin, Inc., New York, Amsterdam, 1964).

[13] C. Itzykson and J.-B. Zuber, Quantum Field Theory (McGraw-Hill, Inc., New York, 1980).

[14] H. Lehmann, K. Symanzik, and W. Zimmermann, Nuovo Cimento 1, 205 (1955).

[15] A. S. Wightman, Phys. Rev. 101, 860 (1956).

[16] H. Lehmann, Nuovo Cimento 10, 579 (1958).

[17] A. Martin, Nuovo Cimento 42, 901 (1966).
[18] R. J. Eden, High Energy Scattering (Cambridge University Press, Cambridge, England, 1967).

[19] R. J. Eden, Rev. Mod. Phys. 43, 15 (1971).

[20] S. M. Roy, Phys. Rep. 5, 125 (1972).

[21] G. Auberson, T. Kinishita, and A. Martin, Phys. Rev. D 3, 3185 (1971).

[22] I. Ya. Pomeranchuk, Zh. Eksperim. i Theor. Fiz. 34, 725 (1958) [Sov. Phys. JETP 7, 499 (1958)], http://www.jetp.ac .ru/cgi-bin/dn/e 00703 0499.pdf.

[23] A. Martin, Nuovo Cimento 39, 704 (1965).

[24] T. Kinoshita, in Perspectives in Modern Physics, edited by R. E. Marshak (Wiley, New York, 1966), p. 211.

[25] A. Martin, Z. Phys. C 15, 185 (1982).

[26] D. A. Fagundes, M. J. Menon, and P. V. R. G. Silva, Braz. J. Phys. 42, 452 (2012).

[27] M. Block and F. Halzen, Braz. J. Phys. 42, 465 (2012).

[28] G. Pancheri and Y. N. Srivastava, Eur. Phys. J. C 77, 150 (2017).

[29] E. Leader, Rev. Mod. Phys. 38, 476 (1966).

[30] W. Rarita, R. J. Riddell, Jr., C. B. Chiu, and R. J. N. Phillips, Phys. Rev. 165, 1615 (1968).

[31] W. Rarita and R. J. N. Phillips, Phys. Rev. Lett. 14, 502 (1965).

[32] L. Baksay et al., Nucl. Phys. B141, 1 (1978); B148, 538(E) (1979).

[33] G. Carboni et al., Phys. Lett. 108B, 145 (1982).

[34] G. Carboni et al., Phys. Lett. 113B, 87 (1982). 
[35] M. Ambrosio et al. (CERN-Napoli-Pisa-Stony Brook Collaboration), Phys. Lett. 113B, 347 (1982).

[36] M. Ambrosio et al. (CERN-Napoli-Pisa-Stony Brook Collaboration), Phys. Lett. 115B, 495 (1982).

[37] G. Carboni et al., Nucl. Phys. B254, 697 (1985).

[38] N. Amos et al., Phys. Lett. 128B, 343 (1983).

[39] D. Favart et al., Phys. Rev. Lett. 47, 1191 (1981).

[40] N. Amos et al., Nucl. Phys. B262, 689 (1985).

[41] N. Amos et al., Phys. Lett. 120B, 460 (1983).

[42] G. Arnison et al. (UA1 Collaboration), Phys. Lett. 128B, 336 (1983).

[43] T. Ekelof, in Proceedings of the International Europhysics Conference on High-Energy Physics, Brighton, 1983 (Rutherford Appleton Laboratory, Chilton, 1983), p. 283 [Report No. CERN-EP-83-146], https://cds.cern.ch/record/ 99443.

[44] R. Battiston et al. (UA4 Collaboration), Phys. Lett. B 117, 126 (1982).

[45] C. Augier et al. (UA4/2 Collaboration), Phys. Lett. B 344, 451 (1995).

[46] M. Bozzo et al. (UA4 Collaboration), Phys. Lett. B 147, 392 (1984).

[47] J. G. Rushbrooke (for UA5 Collaboration), in Proceedings of the 16th International Symposium on Multiparticle Dynamics, Israel, 1985 (World Scientic, Singapore, 1985), p. 289 [Report No. CERN-EP-85-124], http://cds .cern.ch/record/107636.

[48] G. J. Alner et al. (UA5 Collaboration), Z. Phys. C 32, 153 (1986).

[49] F. Abe et al. (CDF Collaboration), Phys. Rev. D 50, 5550 (1994).

[50] S. N. White (for CDF Collaboration), Nucl. Phys. B Proc. Suppl. 25B, 19 (1992).

[51] C. Avila et al. (E-811 Collaboration), Phys. Lett. B 537, 41 (2002).

[52] C. Avila et al. (E-811 Collaboration), Phys. Lett. B 445, 419 (1999).

[53] N. A. Amos et al. (E710 Collaboration), Nuovo Cimento Soc. Ital. Fis. 106A, 123 (1993).

[54] N. A. Amos et al. (E710 Collaboration), Phys. Rev. Lett. 68, 2433 (1992).

[55] N. A. Amos et al. (E710 Collaboration), Phys. Rev. Lett. 63, 2784 (1989).

[56] N. A. Amos et al. (E710 Collaboration), Phys. Lett. B 243, 158 (1990).

[57] G. Aad et al. (ATLAS Collaboration), Nucl. Phys. B889, 486 (2014).

[58] M. Aaboud et al. (ATLAS Collaboration), Phys. Lett. B 761, 158 (2016).

[59] G. Antchev et al. (TOTEM Collaboration), Europhys. Lett. 96, 21002 (2011).
[60] G. Antchev et al. (TOTEM Collaboration), Europhys. Lett. 101, 21002 (2013).

[61] G. Antchev et al. (TOTEM Collaboration), Europhys. Lett. 101, 21004 (2013).

[62] G. Antchev et al. (TOTEM Collaboration), Phys. Rev. Lett. 111, 012001 (2013).

[63] G. Antchev et al. (TOTEM Collaboration), Eur. Phys. J. C 76, 661 (2016).

[64] G. Antchev et al. (TOTEM Collaboration), Eur. Phys. J. C 79, 103 (2019).

[65] Z. Plebaniak and T. Wibig, EPJ Web Conf. 145, 13004 (2017).

[66] R. M. Baltrusaitis, G. L. Cassiday, J. W. Elbert, P. R. Gerhardy, S. Ko, E. C. Loh, Y. Mizumoto, P. Sokolsky, and D. Steck, Phys. Rev. Lett. 52, 1380 (1984).

[67] P. Abreu et al. (Pierre Auger Collaboration), Phys. Rev. Lett. 109, 062002 (2012).

[68] R. U. Abbasi et al. (Telescope Array Collaboration), Phys. Rev. D 92, 032007 (2015).

[69] S. P. Misra and J. Maharana, Phys. Rev. D 14, 133 (1976).

[70] J. D. Talman, Special Functions: A Group Theoretic Approach (Benjamin, New York, 1969), p. 206.

[71] H. Corneille and A. Martin, Nucl. Phys. B115, 163 (1976).

[72] G. Mahoux, Phys. Lett. 65B, 139 (1976).

[73] G. Auberson and S. M. Roy, Nucl. Phys. B117, 322 (1976).

[74] J. Maharana, Commun. Math. Phys. 58, 195 (1978).

[75] J. K. Mohapatra and J. Maharana, Phys. Rev. D 27, 130 (1983).

[76] N. Amos et al., Nucl. Phys. B262, 689 (1985).

[77] M. Bozzo et al. (UA4 Collaboration), Phys. Lett. 147B, 385 (1984).

[78] R. Battiston et al. (UA4 Collaboration), Phys. Lett. 127B, 472 (1983).

[79] D Bernard et al. (UA4 Collaboration), Phys. Lett. B 198, 583 (1987).

[80] A. Abe et al. (CDF Collaboration), Phys. Rev. D 50, 5518 (1994).

[81] N. A. Amos et al. (E710 Collaboration), Phys. Lett. B 247, 127 (1990).

[82] G. Antchev et al. (TOTEM Collaboration), Nucl. Phys. B899, 527 (2015).

[83] G. Antchev et al. (TOTEM Collaboration), Eur. Phys. J. C 79, 861 (2019).

[84] I. M. Dremin and V. A. Nechitailo, Phys. Lett. B 720, 177 (2013).

[85] S. W. MacDowell and A. Martin, Phys. Rev. 135, B960 (1964).

[86] V. Singh, Phys. Rev. Lett. 26, 530 (1971).

[87] M. Block, L. Durand, P. Ha, and F. Halzen, Phys. Rev. D 93, 114009 (2016).

[88] A. Yu. Illarionov, B. A. Kniehl, and A. V. Kotikov, Phys. Rev. Lett. 106, 231802 (2011). 